\title{
Constructing and Refining Computer Science Outreach Focused on Student Engagement
}

\section{Shaya Wolf, University of Wyoming}

Shaya Wolf (swolf4@uwyo.edu) is currently pursuing her Ph.D. at the University of Wyoming. After completing Bachelors degrees in Math and Computer Science, her research focused on distributed systems,industrial control system security, and encryption mechanisms. She is currently working on distributed continuous authentication systems, consensus protocols, and secure embedded intelligence. Her interests also extend to cybersecurity competitions and K12 computer science/cybersecurity outreach and education.

\section{Mr. Rafer Cooley, University of Wyoming}

Rafer Cooley is pursuing a Ph.D. at the University of Wyoming focusing on secure distributed systems. His interests include bio-inspired algorithms, Complex Adaptive Systems, and network protocols. He is currently working on bio-inspired solutions for securing industrial control systems.

Mr. Mason Johnson, University of Wyoming

Dr. Andrea Carneal Burrows, University of Wyoming

Dr. Andrea C. Burrows is an Associate Professor at the University of Wyoming (UW) in the College of Education's (CoEd) School of Teacher Education. She received her doctorate degree from the University of Cincinnati in 2011.She was awarded the UW CoEd Early Career Fellowship (2013), UW CoEd Faculty Award for Outstanding Research and Scholarship (2015), UW CoEd Faculty Award for Outstanding Service to the Education Profession (2016), UW CoEd Honored Fall Convocation Faculty (2017), and UW CoEd Faculty Award for Outstanding Research and Scholarship (2019).Since beginning at UW, Burrows has written, implemented, or evaluated over 50 unique grants. She has been the Program Director for GenCyber as well as PI of NSF grants for STEM and CS work. The core of her research agenda is to deepen science, mathematics, engineering, and technology (STEM) partnership involvement and understanding through STEM interdisciplinary integration with in-service teacher professional development (PD) and pre-service teacher coursework. Her research agenda is composed of a unified STEM education partnership structure and connects educational research to real-world practices.Burrows' many publications appear in leading journals.She is the Co-Editor ofCITE-Journal Science(www.citejournal.org). She is active and presents in several organizations such as AERA, ASEE, ASTE, NSTA, and SITE.Before beginning her work in higher education, she taught secondary school science for 12 years in Florida and Virginia (USA).

\section{Dr. Mike Borowczak, University of Wyoming}

Dr. Mike Borowczak is an Assitant Professor of Computer Science and the Director of the Cybersecurity Education and Research center (CEDAR) at the University of Wyoming. He earned his Ph.D. in Computer Science and Engineering (2013) as well as his BS in Computer Engineering (2007) from the University of Cincinnati. His research focused on detection and prevention of information leakage from hardware side channels. His current research interests include investigating the safety, resilience, and security of decentralized components, devices, and system architectures from theoretical modeling, to simulation and practical implementations. He is also involved in K-20 CS/cybersecurity education research and was the 2019 RMS ASEE conference co-chair.

Mike also has over a decade of industry and research experience - mostly revolving around the semiconductor and bioinformatics industries - with specific experience at Texas Instruments, Intel, and Cincinnati Children's Hospital Medical Center. In addition to his industry experience, Mike spent two years, while completing his Ph.D., as a National Science Foundation GK-12 fellow - teaching and bringing realworld STEM applications in two urban high schools. Since then, he has worked with university faculty to promote and extend K20 STEM outreach in Ohio, Oregon, Texas, and Wyoming. He has authored peer-reviewed articles and papers, presented at national and international conferences, and taught undergraduate/graduate courses in Computer Security, Data Mining, VLSI and pedagogy in STEM. 


\section{Constructing and Refining Engaging Computer Science Outreach}

Shaya Wolf, Rafer Cooley, Mason Johnson, Andrea Burrows, Mike Borowczak

$\{$ swolf4, rcooley2, mjohn107, Andrea.Burrows, Mike.Borowczak\}@uwyo.edu

Abstract \& Motivation
computer science literacy has grown in professional careers. Computer science is integral to problem
solving across the STEM field. Motivated by the need to teach students crucial computer science skills, outreach
camps were held for middle and high school students and specifically focused on cybersecurity. This research
focused on two main questions:
1. How do week-long outreach activities impact student interest in cybersecurity?
2. How do these activities influence student behavior online?

\section{Background}

(2) revious research in this field has shown that:

Computer science should be integrated in STEM fields.

There is a profound need for proper K-12 Computer Science Education

The introduction of computer science education legislation has challenged teachers who are underprepared for instructing computer science.

Outreach camps can have a positive influence on students learning about computer science.

\section{Instruction Techniques}

hese outreach events were focused on maintaining engagement while progressing through a series of computer science and cybersecurity lessons. Researchers favored student-centered, hands-on activities. To gauge the success of the events, learning artifacts were collected from the participants that showed both the qualitative and quantitative results of the sessions. The camps included many different instruction platforms, ranging from class-wide conversations, large group collaboration, small team problem solving, and individual concept interpretation.

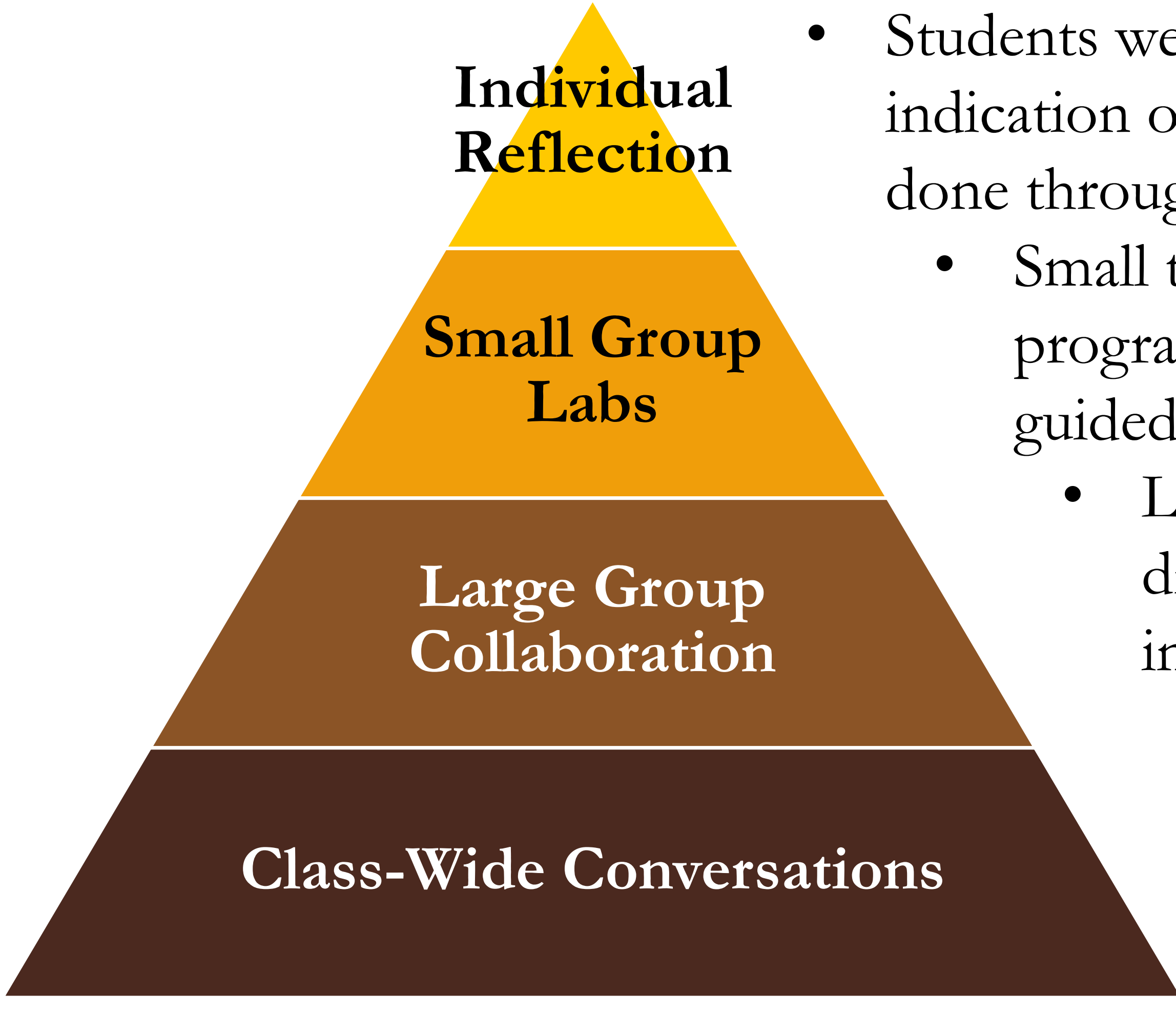

asked to reflect on their learning individually to provide an their progress interest level, and content knowledge. This was rough drawings, worksheets, and surveys. the students were given real world problems then .

Large group collaborations were led by university students. They drove conversations with the participants about notions introduced by the class-wide conversations (5-10 students).

- Class-wide conversations were driven by volunteers with experience in education and focused on introducing main ideas and foundational concepts (25-30 students).

\section{Objectives}

olunteers focused on three main objectives:

1. Engage the participants during sessions through associating lessons with familiar concepts and suitable applications. These real-world connections were made explicitly to the students.

2. Reach for computer science concepts beyond coding. Volunteers developed coursework built on foundational skills such as creative problem solving, communication, teamwork, and critical thinking.

3. Improve instructional techniques throughout the different camps to provide a high-quality educational experience that is up-to-date with current cybersecurity issues.

\section{Results \& Discussion}

D esults were measured using identical pre-surveys and post-surveys that asked the students the same questions before and after the camp activities. This answers our two main research questions.

1 The surveys indicated a rise in interest after

In the surveys, students showed more attention to the

the camp with $65 \%$ reporting high interest.

Interest Level

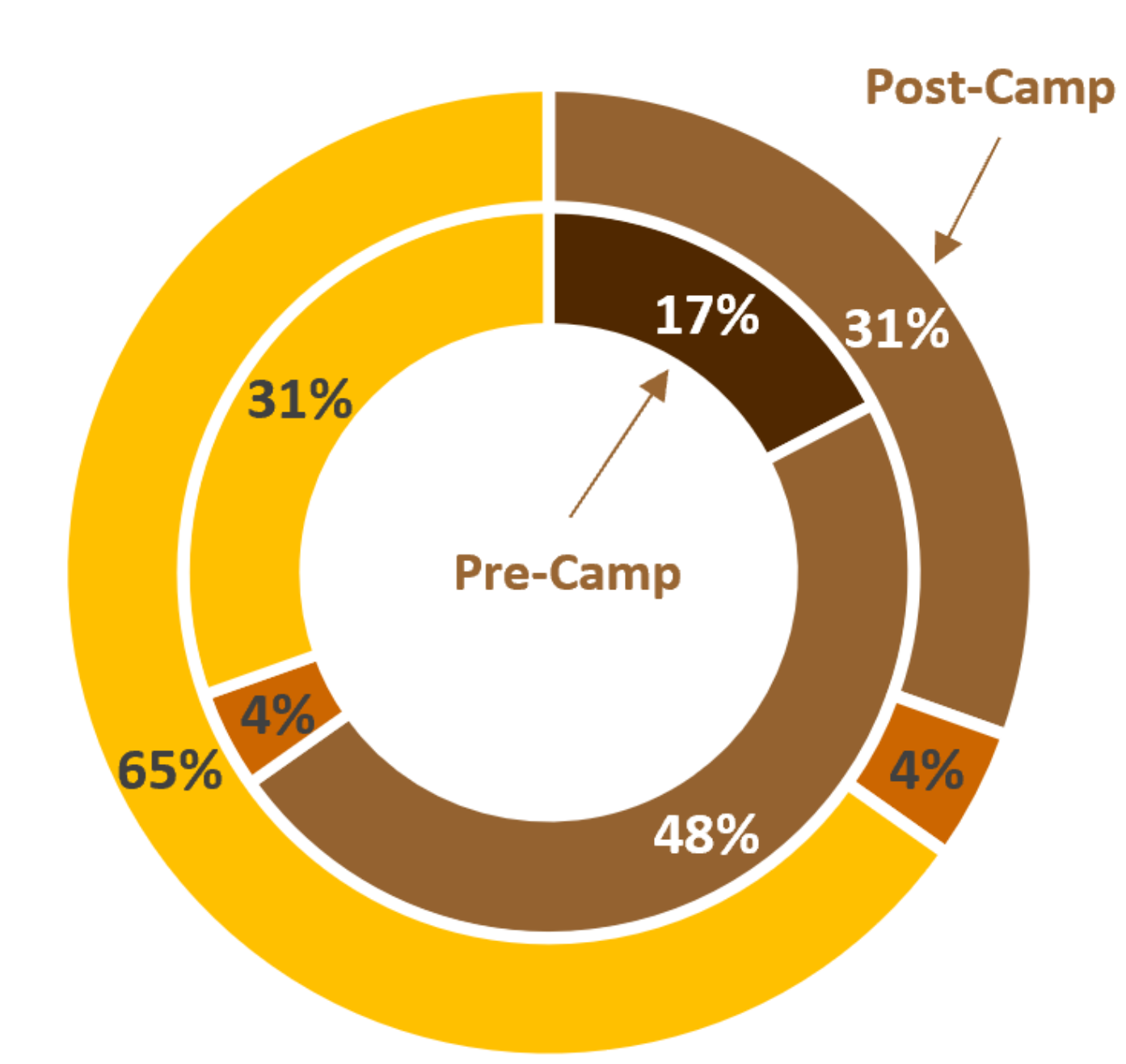

- Minimal | Medium $\quad$ Medium/High $\quad$ Hish implications of their behavior online after the camp.

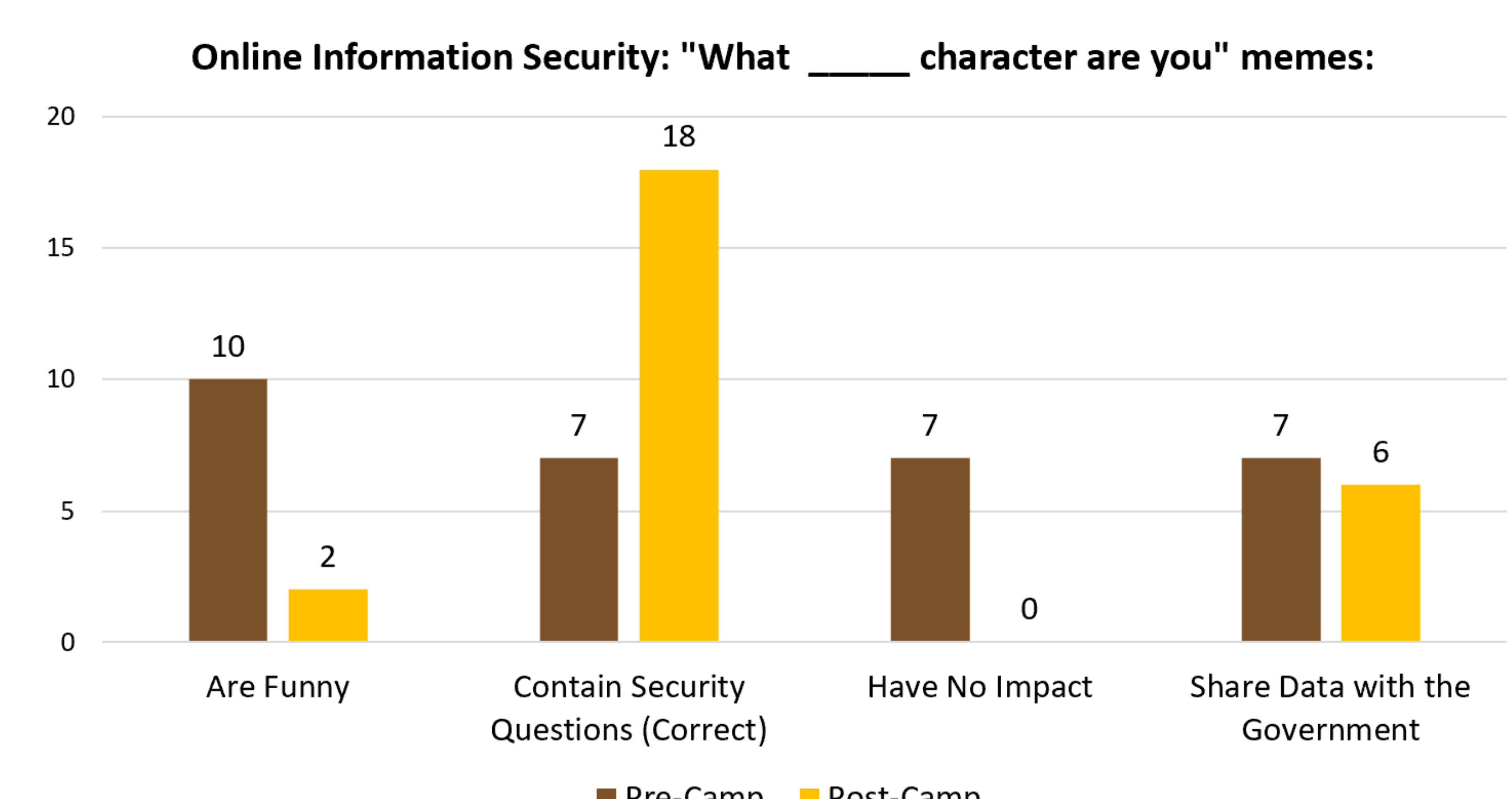

- Pre-Camp - Post-Camp

The volunteers met the objectives for the camps. The activities engaged the participants seen both in the $17.5 \%$ increase in interest level as well as comments from students indicating that they would participate in this camp again. Also, utilizing familiar concepts and suitable applications aided in increasing content knowledge. Further, the coursework was developed with a mix of foundational skills (such as coding) and soft skills (such as problem solving and teamwork). Lastly, using these results, volunteers can enhance future opportunities.

\section{Conclusions \& Future Work}

hese outreach camps have aided in the creation of more outreach opportunities, including additional camps and professional developments for teachers. These include:

Week-long outreach camps for middle and high school students and teachers

- Week-long outreach camps for elementary school students and teachers

Outreach opportunities for teachers to earn micro credentials

1-2 day professional developments to help elementary school teachers meet engineering education standards

-Week-long professional developments to help K-8 teachers integrate computer science lessons in their curriculum

Various professional developments to help teachers intermix computer science lessons into their classrooms

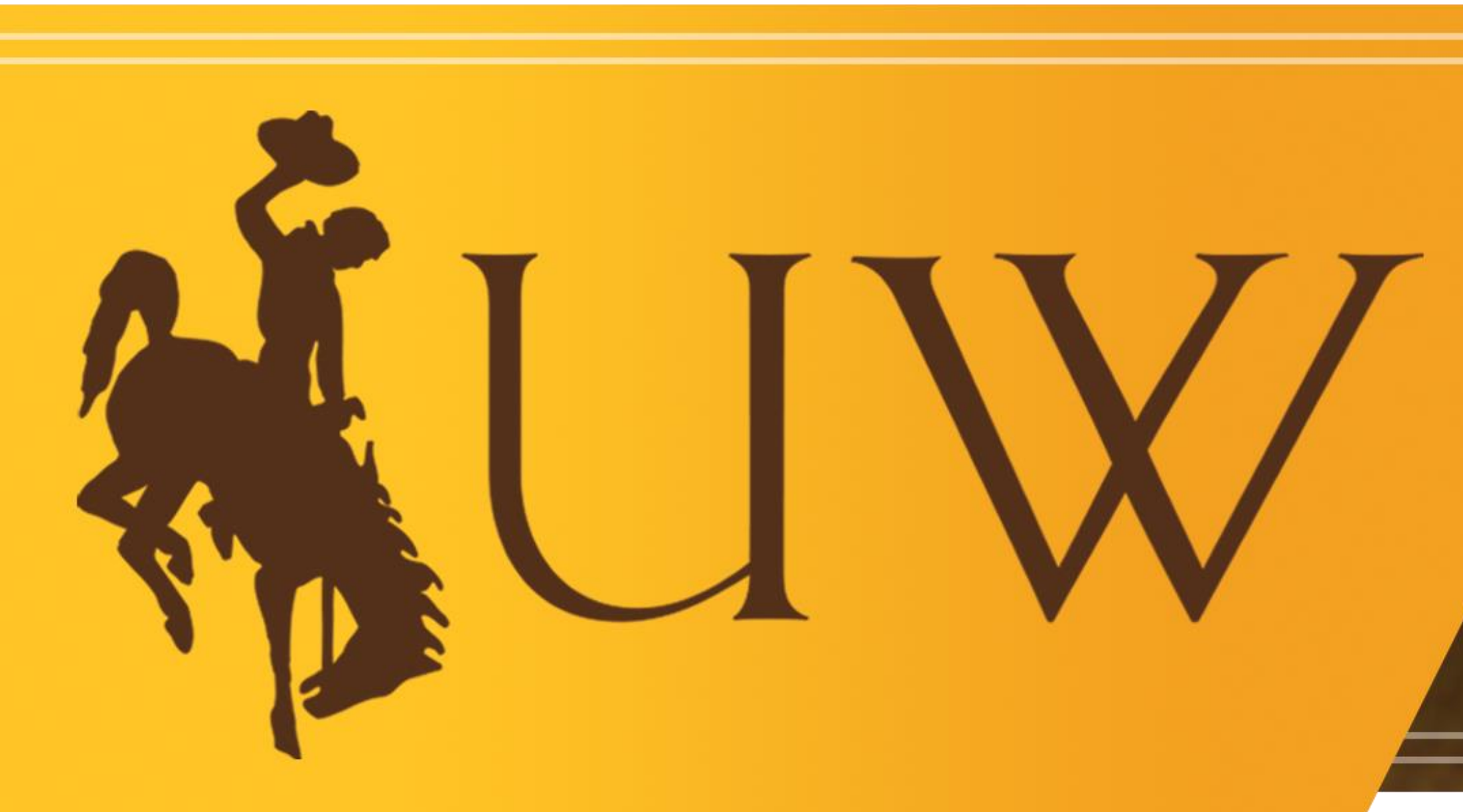

